\title{
A posteriori error covariances in variational data assimilation
}

\author{
V. P. SHUTYAEV*, F.-X. LE DIMET ${ }^{\dagger}$, and I. Yu. GEJADZE
}

\begin{abstract}
The problem of variational data assimilation for a nonlinear evolution model is formulated as an optimal control problem to find some unknown parameters of the model. The equation for the error of the optimal solution is derived through the statistical errors of the input data (background, observation, and model errors). A numerical algorithm is developed to construct an a posteriori covariance operator of the analysis error using the Hessian of an auxiliary optimal control problem based on the tangent linear model constraints.
\end{abstract}

The methods of data assimilation (DA) have become an important tool for analysis of complex physical phenomena in various fields of science and technology. These methods make it possible to combine mathematical models, data resulted from instrumental observations, and a priori information. The problems of variational data assimilation can be formulated as optimal control problems (e.g. [7, 9]) to find unknown model parameters such as initial and/or boundary conditions, righthand sides (forcing), and distributed coefficients. A necessary optimality condition reduces the problem to an optimality system which includes input errors; hence the error in the optimal solution. The statistical properties of the optimal solution error are important for estimating the efficiency of data assimilation in terms of reducing uncertainties in the model parameters and, therefore, in the model output.

The error in the optimal solution can be derived through the errors in the input data using the Hessian of the cost functional of an auxiliary DA problem. For a deterministic case it has been done in [8]. If the errors in the input data are random and subjected to a normal distribution, then for a linearized problem (tangent linear approximation of the model) the covariance matrix of the analysis (optimal estimation of the initial condition) error is given by the inverse of the Hessian matrix of the cost functional (see e.g. [4, 5, 12, 14-16]). This result was given (see e.g. [12]) for a discretized problem. In [3], a similar result was obtained for the continuous operator formulation. We showed that in the nonlinear case a posteriori covariance can often be approximated by the inverse Hessian of the auxiliary control problem ('H-covariance') beyond the validity of the tangent linear hypothesis (TLH).

\footnotetext{
* Institute of Numerical Mathematics, Russian Academy of Sciences, Moscow 119333, Russia

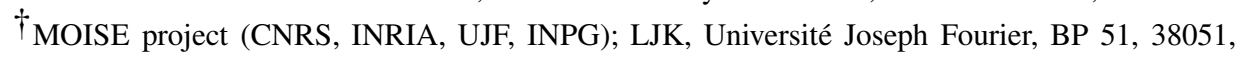
Grenoble Cedex 9, France

\$Department of Civil Engineering, University of Strathclyde, John Anderson Building, 107 Rottenrow, Glasgow, G4 ONG, UK
} 
This paper presents a generalization of the theoretical results reported in [3] to the case of model errors. The equation for the error of the optimal solution is derived through the statistical errors of the input data (background, observation, and model errors). A numerical algorithm is developed to construct an a posteriori covariance operator of the analysis error using the Hessian of an auxiliary optimal control problem based on the tangent linear model constraints. Different approaches to model error formulation in $4 \mathrm{D}$-Var are presented in $[1,17]$ (see also citations in [1]).

The paper is organized as follows. In Section 1, we give the statement of the variational data assimilation problem for a nonlinear evolution model to identify the model parameters. In Section 2, the equation of the error of the optimal solution is derived through the errors of the input data. In Section 3, we derive the formulas and the algorithm for constructing the covariance operator of the optimal solution errors through the covariance operators of the input errors using the Hessian of the cost functional of the auxiliary control problem.

\section{Statement of the problem}

Consider the mathematical model of a physical process that is described by the evolution problem

$$
\left\{\begin{aligned}
\frac{\partial \varphi}{\partial t} & =F(\varphi, \lambda)+f, \quad t \in(0, T) \\
\left.\varphi\right|_{t=0} & =u
\end{aligned}\right.
$$

where $\varphi=\varphi(t)$ is the unknown function belonging for any $t$ to a Hilbert space $X, u \in X, F$ is a nonlinear operator mapping $Y \times Y_{p}$ into $Y$ with $Y=L_{2}(0, T ; X)$, $\|\cdot\|_{Y}=(\cdot, \cdot)_{Y}^{1 / 2}, Y_{p}$ is a Hilbert space (the space of parameters, or control space), $f \in Y$. Suppose that for given $u \in X, f \in Y$ and $\lambda \in Y_{p}$ there exists a unique solution $\varphi \in Y$ to (1.1). The function $\lambda$ is assumed to be an unknown parameter of the model.

Let us introduce the functional

$$
S(\lambda)=\frac{1}{2}\left(V_{1}\left(\lambda-\lambda_{b}\right), \lambda-\lambda_{b}\right)_{Y_{p}}+\frac{1}{2}\left(V_{2}\left(C \varphi-\varphi_{\mathrm{obs}}\right), C \varphi-\varphi_{\mathrm{obs}}\right)_{Y_{\mathrm{obs}}}
$$

where $\lambda_{b} \in Y_{p}$ is a prior (background) function, $\varphi_{\mathrm{obs}} \in Y_{\mathrm{obs}}$ is a prescribed function (observational data), $Y_{\mathrm{obs}}$ is a Hilbert space (observation space), $C: Y \rightarrow Y_{\mathrm{obs}}$ is a linear bounded operator, $V_{1}: Y_{p} \rightarrow Y_{p}$ and $V_{2}: Y_{\mathrm{obs}} \rightarrow Y_{\mathrm{obs}}$ are symmetric positive definite operators.

Consider the following data assimilation problem with the aim to identify the parameter $\lambda$ : find $\lambda \in Y_{p}$ and $\varphi \in Y$ such that they satisfy (1.1), and the functional 
$S(\lambda)$ on the set of solutions to (1.1) takes the minimum value, i.e.

$$
\left\{\begin{aligned}
\frac{\partial \varphi}{\partial t} & =F(\varphi, \lambda)+f, \quad t \in(0, T) \\
\left.\varphi\right|_{t=0} & =u \\
S(\lambda) & =\inf _{v \in Y_{p}} S(v) .
\end{aligned}\right.
$$

We suppose that the solution of (1.3) exists. (Note that the solvability of optimal control parameter estimation problems has been addressed, e.g., in $[2,11]$.) The necessary optimality condition $S^{\prime}(\lambda)=0$ reduces problem (1.3) to the optimality system [13]:

$$
\begin{gathered}
\left\{\begin{array}{c}
\frac{\partial \varphi}{\partial t}=F(\varphi, \lambda)+f, \quad t \in(0, T) \\
\left.\varphi\right|_{t=0}=u
\end{array}\right. \\
\left\{\begin{array}{c}
-\frac{\partial \varphi^{*}}{\partial t}-\left(F_{\varphi}^{\prime}(\varphi, \lambda)\right)^{*} \varphi^{*}=-C^{*} V_{2}\left(C \varphi-\varphi_{\mathrm{obs}}\right), \quad t \in(0, T) \\
\left.\varphi^{*}\right|_{t=T}=0 \\
V_{1}\left(\lambda-\lambda_{b}\right)-\left(F_{\lambda}^{\prime}(\varphi, \lambda)\right)^{*} \varphi^{*}=0 .
\end{array}\right.
\end{gathered}
$$

Here $F_{\varphi}^{\prime}(\varphi, \lambda): Y \rightarrow Y$ and $F_{\lambda}^{\prime}(\varphi, \lambda): Y_{p} \rightarrow Y$ are the Frechet derivatives of $F$ with respect to $\varphi$ and $\lambda$, respectively, $\left(F_{\varphi}^{\prime}(\varphi, \lambda)\right)^{*}: Y \rightarrow Y,\left(F_{\lambda}^{\prime}(\varphi, \lambda)\right)^{*}: Y \rightarrow Y_{p}$ are their adjoints, and $C^{*}$ is the adjoint to $C$ defined by $(C \varphi, \psi)_{Y_{\mathrm{obs}}}=\left(\varphi, C^{*} \psi\right)_{Y}, \varphi \in Y, \psi \in$ $Y_{\text {obs. }}$.

We assume that system (1.4)-(1.6) has a unique solution. Suppose that $\lambda_{b}=$ $\bar{\lambda}+\xi_{1}, \varphi_{\mathrm{obs}}=C \bar{\varphi}+\xi_{2}, f=\bar{f}+\xi_{3}$, where $\xi_{1} \in Y_{p}, \xi_{2} \in Y_{\mathrm{obs}}, \xi_{3} \in Y$, and $\bar{\varphi}$ is the ('true') solution to the problem (1.1) with $\lambda=\bar{\lambda}$ and $f=\bar{f}$ :

$$
\left\{\begin{aligned}
\frac{\partial \bar{\varphi}}{\partial t} & =F(\bar{\varphi}, \bar{\lambda})+\bar{f}, \quad t \in(0, T) \\
\left.\bar{\varphi}\right|_{t=0} & =\bar{u} .
\end{aligned}\right.
$$

The functions $\xi_{1}, \xi_{2}, \xi_{3}$ are treated as the errors of the input data $\lambda_{b}, \varphi_{\mathrm{obs}}, f$ ('background', observation, and model errors, respectively). For $V_{1}$ and $V_{2}$ in (1.2), one usually has $V_{1}=V_{\xi_{1}}^{-1}, V_{2}=V_{\xi_{2}}^{-1}$, where $V_{\xi}$ is the covariance operator of the corresponding error $\xi$.

\section{Equation for the optimal solution error}

Let us derive the equation for the optimal solution error through input errors. Let $\delta \varphi=\varphi-\bar{\varphi}, \delta \lambda=\lambda-\bar{\lambda}$. Then, from (1.7) and the optimality system (1.4)-(1.6), we obtain 


$$
\begin{aligned}
& \begin{array}{rllllllll}
\frac{\partial \delta \varphi}{\partial t} & F_{\varphi} \tilde{\varphi} \tilde{\lambda} \delta \varphi & F_{\lambda} \tilde{\varphi} \tilde{\lambda} \delta \lambda & \xi_{3} & t & 0, T) \\
& & \delta \varphi_{t} 0 & 0 & & &
\end{array} \\
& \left.\frac{\partial \varphi}{\partial t} \quad F_{\varphi} \varphi \lambda \underset{\left.\varphi^{*}\right|_{t=T}=0}{\varphi} C V_{2} C \delta \varphi \quad \xi_{2} \quad t \quad 0, T\right) \\
& V_{1}\left(\delta \lambda-\xi_{1}\right)-\left(F_{\lambda}^{\prime}(\varphi, \lambda)\right)^{*} \varphi^{*}=0
\end{aligned}
$$

where $\tilde{\varphi}=\bar{\varphi}+\tau(\varphi-\bar{\varphi}), \tilde{\lambda}=\bar{\lambda}+\tau(\lambda-\bar{\lambda}) \tau \in[0,1]$.

Note that $\tilde{\varphi}=\bar{\varphi}+\tau \delta \varphi, \varphi=\bar{\varphi}+\delta \varphi, \tilde{\lambda}=\bar{\lambda}+\tau \delta \lambda, \lambda=\bar{\lambda}+\delta \lambda$. The system (2.1)-(2.3) can be written in the form:

$$
\begin{gathered}
\left\{\begin{aligned}
\frac{\partial \delta \varphi}{\partial t}-F_{\varphi}^{\prime}(\bar{\varphi}, \bar{\lambda}) \delta \varphi & =F_{\lambda}^{\prime}(\bar{\varphi}, \bar{\lambda}) \delta \lambda+\xi_{3}+\tilde{\xi}_{3}, \quad t \in(0, T) \\
\left.\delta \varphi\right|_{t=0} & =0
\end{aligned}\right. \\
\left\{\begin{aligned}
&-\frac{\partial \varphi^{*}}{\partial t}-\left(F_{\varphi}^{\prime}(\bar{\varphi}, \bar{\lambda})\right)^{*} \varphi^{*}=-C^{*} V_{2}\left(C \delta \varphi-\xi_{2}\right)+\xi_{4}, \quad t \in(0, T) \\
&\left.\varphi^{*}\right|_{t=T}=0 \\
& V_{1}\left(\delta \lambda-\xi_{1}\right)-\left(F_{\lambda}^{\prime}(\bar{\varphi}, \bar{\lambda})\right)^{*} \varphi^{*}=\xi_{5}
\end{aligned}\right.
\end{gathered}
$$

where

$$
\tilde{\xi}_{3}=\left[F_{\varphi}^{\prime}(\tilde{\varphi}, \tilde{\lambda})-F_{\varphi}^{\prime}(\bar{\varphi}, \bar{\lambda})\right] \delta \varphi+\left[F_{\lambda}^{\prime}(\tilde{\varphi}, \tilde{\lambda})-F_{\lambda}^{\prime}(\bar{\varphi}, \bar{\lambda})\right] \delta \lambda
$$

$$
\xi_{4}=\left[\left(F_{\varphi}^{\prime}(\varphi, \lambda)\right)^{*}-\left(F_{\varphi}^{\prime}(\bar{\varphi}, \bar{\lambda})\right)^{*}\right] \varphi^{*}, \quad \xi_{5}=\left[\left(F_{\lambda}^{\prime}(\varphi, \lambda)\right)^{*}-\left(F_{\lambda}^{\prime}(\bar{\varphi}, \bar{\lambda})\right)^{*}\right] \varphi^{*}
$$

Let us introduce the operator $H: Y_{p} \rightarrow Y_{p}$ defined by the successive solutions of the following problems:

$$
\begin{gathered}
\left\{\begin{array}{c}
\frac{\partial \psi}{\partial t}-F_{\varphi}^{\prime}(\bar{\varphi}, \bar{\lambda}) \psi=F_{\lambda}^{\prime}(\bar{\varphi}, \bar{\lambda}) v, \quad t \in(0, T) \\
\left.\psi\right|_{t=0}=0
\end{array}\right. \\
\left\{\begin{array}{c}
-\frac{\partial \psi^{*}}{\partial t}-\left(F_{\varphi}^{\prime}(\bar{\varphi}, \bar{\lambda})\right)^{*} \psi^{*}=-C^{*} V_{2} C \psi, \quad t \in(0, T) \\
\left.\psi^{*}\right|_{t=T}=0 \\
H v=V_{1} v-\left(F_{\lambda}^{\prime}(\bar{\varphi}, \bar{\lambda})\right)^{*} \psi^{*} .
\end{array}\right.
\end{gathered}
$$

Below we introduce four auxiliary operators $R_{1}, R_{2}, R_{3}, R_{4}$. Let $R_{1}=V_{1}$. Let us introduce the operator $R_{2}: Y_{\mathrm{obs}} \rightarrow Y_{p}$ acting on the functions $g \in Y_{\mathrm{obs}}$ according to the formula

$$
R_{2} g=\left(F_{\lambda}^{\prime}(\bar{\varphi}, \bar{\lambda})\right)^{*} \theta^{*}
$$


where $\theta^{*}$ is the solution to the adjoint problem

$$
\left\{\begin{aligned}
-\frac{\partial \theta^{*}}{\partial t}-\left(F_{\varphi}^{\prime}(\bar{\varphi}, \bar{\lambda})\right)^{*} \theta^{*} & =C^{*} V_{2} g, \quad t \in(0, T) \\
\left.\theta^{*}\right|_{t=T} & =0 .
\end{aligned}\right.
$$

The operator $R_{3}: Y \rightarrow Y_{p}$ is defined on the functions $q \in Y$ as follows:

$$
\begin{gathered}
\left\{\begin{aligned}
\frac{\partial \theta_{1}}{\partial t}-F_{\varphi}^{\prime}(\bar{\varphi}, \bar{\lambda}) \theta_{1} & =q, \quad t \in(0, T) \\
\left.\theta_{1}\right|_{t=0} & =0
\end{aligned}\right. \\
\left\{\begin{array}{c}
-\frac{\partial \theta_{1}^{*}}{\partial t}-\left(F_{\varphi}^{\prime}(\bar{\varphi}, \bar{\lambda})\right)^{*} \theta_{1}^{*}=-C^{*} V_{2} C \theta_{1}, \quad t \in(0, T) \\
\left.\theta_{1}^{*}\right|_{t=T}=0 \\
R_{3} q=\left(F_{\lambda}^{\prime}(\bar{\varphi}, \bar{\lambda})\right)^{*} \theta_{1}^{*} .
\end{array}\right.
\end{gathered}
$$

The operator $R_{4}: Y \rightarrow Y_{p}$ is defined on the functions $h \in Y$ as

$$
\begin{gathered}
-\frac{\partial \theta_{2}^{*}}{\partial t}-\left(F_{\varphi}^{\prime}(\bar{\varphi}, \bar{\lambda})\right)^{*} \theta_{2}^{*}=h, \quad t \in(0, T) \\
\left.\theta_{1}^{*}\right|_{t=T}=0 \\
R_{4} h=\left(F_{\lambda}^{\prime}(\bar{\varphi}, \bar{\lambda})\right)^{*} \theta_{2}^{*} .
\end{gathered}
$$

From (2.7)-(2.16) we conclude that system (2.4)-(2.6) is equivalent to the single equation for $\delta \lambda$ :

$$
H \delta \lambda=R_{1} \xi_{1}+R_{2} \xi_{2}+R_{3}\left(\xi_{3}+\tilde{\xi}_{3}\right)+R_{4} \xi_{4}+\xi_{5}
$$

This is the exact equation for $\delta \lambda$. Under the hypothesis that $H$ is invertible, we get

$$
\delta \lambda=T_{1} \xi_{1}+T_{2} \xi_{2}+T_{3}\left(\xi_{3}+\tilde{\xi}_{3}\right)+T_{4} \xi_{4}+T_{5} \xi_{5}
$$

where $T_{i}=H^{-1} R_{i}, i=1,2,3,4, T_{5}=H^{-1}, T_{1}: Y_{p} \rightarrow Y_{p}, T_{2}: Y_{\mathrm{obs}} \rightarrow Y_{p}, T_{3}, T_{4}: Y \rightarrow Y_{p}$.

Note, however, that the functions $\varphi, \lambda, \tilde{\varphi}, \tilde{\lambda}$ in (2.1)-(2.3) depend on $\xi_{1}, \xi_{2}, \xi_{3}$, as the result, the terms $T_{3} \xi_{3}, T_{4} \xi_{4}, T_{5} \xi_{5}$ also depend on $\xi_{1}, \xi_{2}, \xi_{3}$ (nonlinearly), and it is not possible to represent $\delta \lambda$ through $\xi_{1}, \xi_{2}, \xi_{3}$ in an explicit form. To derive from (2.18) the covariance operator of $\delta \lambda$, we need to introduce some approximation of (2.18). Since $\tilde{\varphi}=\bar{\varphi}+\tau \delta \varphi, \varphi=\bar{\varphi}+\delta \varphi, \tilde{\lambda}=\bar{\lambda}+\tau \delta \lambda, \lambda=\bar{\lambda}+\delta \lambda$, we assume that

$$
T_{3} \tilde{\xi}_{3} \approx 0, \quad T_{4} \xi_{4} \approx 0, \quad T_{5} \xi_{5} \approx 0
$$

then (2.18) reduces to

$$
\delta \lambda=T_{1} \xi_{1}+T_{2} \xi_{2}+T_{3} \xi_{3}
$$

which is equivalent to the system: 


$$
\begin{gathered}
\left\{\begin{aligned}
\frac{\partial \delta \varphi}{\partial t}-F_{\varphi}^{\prime}(\bar{\varphi}, \bar{\lambda}) \delta \varphi & =F_{\lambda}^{\prime}(\bar{\varphi}, \bar{\lambda}) \delta \lambda+\xi_{3}, \quad t \in(0, T) \\
\left.\delta \varphi\right|_{t=0} & =0
\end{aligned}\right. \\
\left\{\begin{aligned}
-\frac{\partial \varphi^{*}}{\partial t}-\left(F_{\varphi}^{\prime}(\bar{\varphi}, \bar{\lambda})\right)^{*} \varphi^{*} & =-C^{*} V_{2}\left(C \delta \varphi-\xi_{2}\right), \quad t \in(0, T) \\
\left.\varphi^{*}\right|_{t=T} & =0 \\
V_{1}\left(\delta \lambda-\xi_{1}\right) & -\left(F_{\lambda}^{\prime}(\bar{\varphi}, \bar{\lambda})\right)^{*} \varphi^{*}=0 .
\end{aligned}\right.
\end{gathered}
$$

Taking into account the definition of $\tilde{\xi}_{3}, \xi_{4}, \xi_{5}$, it is easily seen that assumption (2.19) comes from the first-order approximation of the Taylor-Lagrange formula under the hypothesis that $F$ is twice continuously Frechet differentiable [10]. Using this formula, the errors $\tilde{\xi}_{3}, \xi_{4}, \xi_{5}$, may be expressed through the second derivatives of $F$, and the values of the norms of $T_{3} \tilde{\xi}_{3}, T_{4} \xi_{4}, T_{5} \xi_{5}$ can be estimated, thus giving the possibility to analyse the approximation error when taking (2.20) instead of (2.18).

The problem (2.21)-(2.23) is a linear data assimilation problem; for fixed $\bar{\lambda}, \bar{\varphi}$ it is the necessary optimality condition to the following (auxiliary) minimization problem: Find $\delta \lambda$ and $\delta \varphi$ such that

$$
\left\{\begin{aligned}
\frac{\partial \delta \varphi}{\partial t}-F_{\varphi}^{\prime}(\bar{\varphi}, \bar{\lambda}) \delta \varphi & =F_{\lambda}^{\prime}(\bar{\varphi}, \bar{\lambda}) \delta \lambda+\xi_{3}, \quad t \in(0, T) \\
\left.\delta \varphi\right|_{t=0} & =0 \\
S_{1}(\delta \lambda) & =\inf _{v \in Y_{p}} S_{1}(v)
\end{aligned}\right.
$$

where

$$
S_{1}(\delta \lambda)=\frac{1}{2}\left(V_{1}\left(\delta \lambda-\xi_{1}\right), \delta \lambda-\xi_{1}\right)_{Y_{p}}+\frac{1}{2}\left(V_{2}\left(C \delta \varphi-\xi_{2}\right), C \delta \varphi-\xi_{2}\right)_{Y_{\mathrm{obs}}} .
$$

The Hessian $H$ of functional (2.25) is defined on $v \in Y_{p}$ by (2.7)-(2.9). Note that for $\xi_{2}=0$ the operator $H$ coincides with the Hessian $\mathscr{H}$ of the original nonlinear DA problem on the exact solution $\bar{\lambda}$. The Hessian $H$ acts in $Y_{p}$ as a self-adjoint operator with the domain of definition $D(H)=Y_{p}$. Moreover, due to $V_{1}, V_{2}$, the operator $H$ is positive definite, and hence invertible.

Note that if the tangent linear hypothesis is valid (e.g. [4]), then for small $\delta \varphi, \delta \lambda$ we can choose (2.19). However, the transition from (2.18) to (2.20) may not necessarily require the tangent linear hypothesis to be valid.

As follows from (2.20), the influence of the errors $\xi_{1}, \xi_{2}, \xi_{3}$ on the value of the error $\delta \lambda$ of the optimal solution is determined by the operators $H^{-1} R_{1}, H^{-1} R_{2}$, $H^{-1} R_{3}$, respectively. The values of the norms of these operators can be considered as sensitivity coefficients: the less is the norm of the operator $H^{-1} R_{i}$, the less impact on $\delta \lambda$ is given by the corresponding error $\xi_{i}$. This criterion was used for deterministic error analysis in $[6,8]$ with the aim to identify the initial condition. Here, assuming 
the statistical structure of the errors $\xi_{1}, \xi_{2}, \xi_{3}$, we will derive the covariance operator of the optimal solution (parameter) error through the covariance operators of the input errors and develop a numerical algorithm to construct the covariance operator of the optimal solution error using the covariance operators of the input errors.

\section{Covariance operator as the inverse Hessian}

Consider error equation (2.20), where $T_{i}=H^{-1} R_{i}, i=1,2,3, T_{1}: Y_{p} \rightarrow Y_{p}$, $T_{2}: Y_{\mathrm{obs}} \rightarrow Y_{p}, T_{3}: Y \rightarrow Y_{p}$. Below we suppose that the errors $\xi_{1}, \xi_{2}, \xi_{3}$ are normally distributed, unbiased, and mutually uncorrelated. By $V_{\xi_{i}}$ we denote the covariance operator of the corresponding error $\xi_{i}, i=1,2,3$, i.e. $V_{\xi_{1}} \cdot=E\left[\left(\cdot, \xi_{1}\right)_{Y_{p}} \xi_{1}\right]$, $V_{\xi_{2}} \cdot=E\left[\left(\cdot, \xi_{2}\right)_{Y_{\mathrm{obs}}} \xi_{2}\right], V_{\xi_{3}} \cdot=E\left[\left(\cdot, \xi_{3}\right)_{Y} \xi_{3}\right]$, where $E$ is the expectation. By $V_{\delta \lambda}$ we denote the covariance operator of the optimal solution (analysis) error: $V_{\delta \lambda} \cdot=$ $E\left[(\cdot, \delta \lambda)_{Y_{p}} \delta \lambda\right]$. From (2.20) we get

$$
V_{\delta \lambda}=T_{1} V_{\xi_{1}} T_{1}^{*}+T_{2} V_{\xi_{2}} T_{2}^{*}+T_{3} V_{\xi_{3}} T_{3}^{*} .
$$

To find the covariance operator $V_{\delta \lambda}$, we need to construct the operators $T_{i} V_{\xi_{i}} T_{i}^{*}$, $i=1,2,3$. We proved in [13] that

$$
T_{1} V_{\xi_{1}} T_{1}^{*}+T_{2} V_{\xi_{2}} T_{2}^{*}=H^{-1}
$$

where $H$ is the Hessian of the auxiliary data assimilation problem (2.24)-(2.25) defined by (2.7)-(2.9). Then,

$$
V_{\delta \lambda}=H^{-1}+T_{3} V_{\xi_{3}} T_{3}^{*} .
$$

Consider now the operator $T_{3}=H^{-1} R_{3}$. To construct $T_{3} V_{\xi_{3}} T_{3}^{*}$, we need to derive $R_{3}^{*}$. For $q \in Y, p \in Y_{p}$, we have from (2.12)-(2.14):

$$
\left(R_{3} q, p\right)_{Y_{p}}=\left(\left(F_{\lambda}^{\prime}(\bar{\varphi}, \bar{\lambda})\right)^{*} \theta_{1}^{*}, p\right)_{Y_{p}}=-\left(C^{*} V_{2} C \theta_{1}, \phi\right)_{Y}=-\left(V_{2} C \theta_{1}, C \phi\right)_{Y_{\mathrm{obs}}}
$$

where $\theta_{1}, \theta_{1}^{*}$ are the solutions to (2.12)-(2.13), and $\phi$ is the solution to (2.7) for $v=p$. Further,

$$
\left(R_{3} q, p\right)_{Y_{p}}=-\left(\theta_{1}, C^{*} V_{2} C \phi\right)_{Y}=\left(q, \phi^{*}\right)_{Y}
$$

and $R_{3}^{*} p=\phi^{*}$, where $\phi^{*}$ is the solution to the adjoint problem:

$$
\left\{\begin{aligned}
-\frac{\partial \phi^{*}}{\partial t}-\left(F_{\varphi}^{\prime}(\bar{\varphi}, \bar{\lambda})\right)^{*} \phi^{*} & =-C^{*} V_{2} C \phi, \quad t \in(0, T) \\
\left.\phi^{*}\right|_{t=T} & =0 .
\end{aligned}\right.
$$

Let $Q=R_{3} V_{\xi_{3}} R_{3}^{*}: Y_{p} \rightarrow Y_{p}$. The operator $Q$ can be defined as follows: for a given $p \in Y_{p}$ find $\phi$ as the solution of (2.7) for $v=p$, find $\phi^{*}$ as the solution of (3.4), and for $q=V_{\xi_{3}} \phi^{*}$ find $\theta_{1}, \theta_{1}^{*}$ as the solutions of (2.12)-(2.13); then put $R_{3} V_{\xi_{3}} R_{3}^{*}=$ $\left(F_{\lambda}^{\prime}(\bar{\varphi}, \bar{\lambda})\right)^{*} \theta_{1}^{*}$. 
Therefore, $T_{3} V_{\xi_{3}} T_{3}^{*}=H^{-1} R_{3} V_{\xi_{3}} R_{3}^{*} H^{-1}=H^{-1} Q H^{-1}$, and $Q$ is defined by the successive solutions of the following problems (for the given $p \in Y_{p}$ ):

$$
\begin{gathered}
\left\{\begin{aligned}
& \frac{\partial \phi}{\partial t}-F_{\varphi}^{\prime}(\bar{\varphi}, \bar{\lambda}) \phi= F_{\lambda}^{\prime}(\bar{\varphi}, \bar{\lambda}) p, \quad t \in(0, T) \\
&\left.\phi\right|_{t=0}=0
\end{aligned}\right. \\
\left\{\begin{aligned}
-\frac{\partial \phi^{*}}{\partial t}-\left(F_{\varphi}^{\prime}(\bar{\varphi}, \bar{\lambda})\right)^{*} \phi^{*} & =-C^{*} V_{2} C \phi, \quad t \in(0, T) \\
\left.\phi^{*}\right|_{t=T} & =0
\end{aligned}\right. \\
\left\{\begin{aligned}
\frac{\partial \theta_{1}}{\partial t}-F_{\varphi}^{\prime}(\bar{\varphi}, \bar{\lambda}) \theta_{1} & =V_{\xi_{3}} \phi^{*}, \quad t \in(0, T) \\
\left.\theta_{1}\right|_{t=0} & =0
\end{aligned}\right. \\
\left\{\begin{aligned}
-\frac{\partial \theta_{1}^{*}}{\partial t}-\left(F_{\varphi}^{\prime}(\bar{\varphi}, \bar{\lambda})\right)^{*} \theta_{1}^{*} & =-C^{*} V_{2} C \theta_{1}, \quad t \in(0, T) \\
\left.\theta_{1}^{*}\right|_{t=T} & =0
\end{aligned}\right.
\end{gathered}
$$

then

$$
Q p=\left(F_{\lambda}^{\prime}(\bar{\varphi}, \bar{\lambda})\right)^{*} \theta_{1}^{*}
$$

The algorithm (3.5)-(3.9) can be used to compute the operator $Q$ numerically. Then, from (3.3), we come to the main result of the paper.

Theorem 3.1. The covariance operator $V_{\delta \lambda}$ of the optimal solution error is given by the formula

$$
V_{\delta \lambda}=H^{-1}+H^{-1} Q H^{-1}
$$

where $H$ is the Hessian of the functional $S_{1}$ defined by (2.7)-(2.9), and the operator $Q$ is defined by (3.5)-(3.9).

It is not difficult to show that $Q$ is the Hessian of the following minimization problem: Find $v \in Y_{p}$ such that

$$
S_{Q}(v)=\inf _{p \in Y_{p}} S_{Q}(p)
$$

where

$$
S_{Q}(p)=\frac{1}{2}\left(V_{\xi_{3}} \phi^{*}, \phi^{*}\right)_{Y}
$$

and $\phi^{*}$ is defined through $p$ by the successive solutions of the following problems:

$$
\begin{aligned}
& \left\{\begin{aligned}
\frac{\partial \phi}{\partial t}-F_{\varphi}^{\prime}(\bar{\varphi}, \bar{\lambda}) \phi & =F_{\lambda}^{\prime}(\bar{\varphi}, \bar{\lambda}) p, \quad t \in(0, T) \\
\left.\phi\right|_{t=0} & =0
\end{aligned}\right. \\
& \left\{\begin{aligned}
-\frac{\partial \phi^{*}}{\partial t}-\left(F_{\varphi}^{\prime}(\bar{\varphi}, \bar{\lambda})\right)^{*} \phi^{*} & =-C^{*} V_{2} C \phi, \quad t \in(0, T) \\
\left.\phi^{*}\right|_{t=T} & =0 .
\end{aligned}\right.
\end{aligned}
$$




\section{Acknowledgements}

This work was carried out within the MOISE project (CNRS, INRIA, UJF, INPG), the ADAMS project, and project 06-01-00344 of the Russian Foundation for Basic Research and within the program ECO-NET (EGIDE).

\section{References}

1. S. Akella and I. M. Navon, Different approaches to model error formulation in 4D-Var: a study with high resolution advection schemes. Tellus A (2009), 61A, 112-128.

2. G. Chavent, Local stability of the output least square parameter estimation technique. Math. Appl. Comp. (1983) 2, 3-22.

3. I. Gejadze, F.-X. Le Dimet, and V. Shutyaev, On analysis error covariances in variational data assimilation. SIAM J. Sci. Comp. (2008) 30, No. 4, 1847-1874.

4. J. R. Gunson and P. Malanotte-Rizzoli, Assimilation studies of open-ocean flows, 2. Error measures with strongly nonlinear dynamics. J. Geophys. Res. (1996) 101, No. C12, 28473-28488.

5. F.-X. Le Dimet, I. M. Navon, and D. N. Daescu, Second-order information in data assimilation. Monthly Weather Review (2002) 130, No. 3, 629-648.

6. F.-X. Le Dimet, P. Ngnepieba, and V. Shutyaev, On error analysis in data assimilation problems. Russ. J. Numer. Anal. Math. Modelling (2002) 17, No. 1, 71-97.

7. F.-X. Le Dimet and O. Talagrand, Variational algorithms for analysis and assimilation of meteorological observations: theoretical aspects. Tellus (1986) 38A, 97-110.

8. F.-X. Le Dimet and V. Shutyaev, On deterministic error analysis in variational data assimilation. Nonlinear Processes in Geophysics (2005) 14, 1-10.

9. J.-L. Lions, Contrôle optimal des systèmes gouvernés par des équations aux dérivées partielles. Dunod, Paris, 1968.

10. G. I. Marchuk, V. I. Agoshkov, and V. P. Shutyaev, Adjoint Equations and Perturbation Algorithms in Nonlinear Problems. CRC Press Inc., New York, 1996.

11. I. M. Navon, Practical and theoretical aspects of adjoint parameter estimation and identifiability in meteorology and oceanography. Dynamics of Atmospheres and Oceans. Special issue in honor of Richard Pfeffer, 1997, Vol. 27, pp. 55-79.

12. F. Rabier and P. Courtier, Four-dimensional assimilation in the presence of baroclinic instability. Quart. J. Roy. Meteorol. Soc. (1992) 118, 649-672.

13. V. Shutyaev, F.-X. Le Dimet, I. Gejadze, On optimal solution error covariances in variational data assimilation. Russ. J. Numer. Anal. Math. Modelling (2008) 23, No. 2, 197-205.

14. W. C. Thacker, The role of the Hessian matrix in fitting models to measurements. J. Geophys. Res. (1989) 94, No. C5, 6177-6196.

15. J. N. Thepaut and P. Courtier, Four-dimensional variational assimilation using the adjoint of a multilevel primitive equation model. Quart. J. Roy. Meteorol. Soc. (1991) 117, 1225-1254.

16. F. Veersé, Variable-storage quasi-Newton operators as inverse forecast/analysis error covariance matrices in variational data assimilation. INRIA Tech. Report, 1999, No. 3685.

17. P. A. Vidard, A. Piacentini, and F.-X. Le Dimet, Variational data analysis with control of the forecast bias. Tellus A (2004) 56, No. 3, 177-188. 
